\title{
Diferenças na Detecção de Frequências Espaciais e Radiais em Crianças ${ }^{1}$
}

\author{
Natanael Antonio dos Santos ${ }^{2}$ \\ Universidade Federal da Paraíba \\ Valtenice de Cássia Rodrigues de Matos França \\ Universidade de São Paulo \\ Príscilla Anny de Araújo Alves \\ Universidade Federal da Paraíba
}

\begin{abstract}
RESUMO - O objetivo deste estudo foi comparar curvas de sensibilidade ao contraste para estímulos radiais (FSCr) e grades senoidais (FSC) de 0,25, 0,5, 1 e 2 cpg em crianças de 6 a 13 anos. Foram mensurados limiares de contraste para 40 crianças, utilizando o método psicofísico da escolha forçada e níveis baixos de luminância. Todas estavam livres de doenças oculares e tinham acuidade visual normal. Os resultados mostraram que a sensibilidade das crianças foi maior para grades senoidais (FSC) do que para estímulos radiais (FSCr). Esses resultados sugerem que esses estímulos podem ser processados por áreas visuais distintas.
\end{abstract}

Palavras-chave: percepção visual; sensibilidade ao contraste; desenvolvimento visual; frequência radial e espacial; método da escolha forçada.

\section{Differences in Detection of Spatial and Radial Frequencies in Children}

\begin{abstract}
The aim of this work was to compare contrast sensitivity curves for radial (rCSF) and sine-wave gratings (CSF) stimuli of $0.25,0.5,1$ and 2 cpd in 6 to 13 year-old-children. It was measured the contrast thresholds of 40 children using the psychophysical forced-choice method and low luminance levels. All children were free of identifiable ocular diseases and had normal acuity. The results showed that contrast sensitivity of the children was higher for spatial frequencies (CSF) than radial frequencies (rCSF). The results suggest that these stimuli can be processed by different visual cortical areas.
\end{abstract}

Keywords: visual perception; contrast sensitivity; visual development; radial and spatial frequency; forced-choice method.

A função de sensibilidade ao contraste (FSC) é uma medida clássica definida por Cornsweet (1970) como o inverso da curva de limiar de contraste (1/FSC). Nesses termos, o limiar de contraste (ou sensibilidade de contraste) trata da menor quantidade de variação de luminância que o sistema visual humano precisa para detectar um determinado padrão ou frequência espacial. Assim, na mensuração da sensibilidade ao contraste, as variáveis experimentais principais são a frequência espacial (variável independente) e o contraste (variável dependente). Frequência espacial é o número de ciclos por grau de ângulo visual. O contraste é a relação entre a diferença da luminância máxima e mínima dividida pela soma da luminância máxima e mínima.

A FSC permite caracterizar o desempenho ou a resposta do sistema visual para padrões espaciais em níveis baixos, médios e altos de contraste, e avaliar possíveis alterações no curso natural do desenvolvimento do sistema visual (Candy, Crowell \& Banks, 1998; Van Sluyters, Atkinson, Held, Hoffman \& Shatz, 1990) ou nas vias e mecanismos senso-

1 Trabalho apresentado na XXI Reunião Anual da Federação de Sociedades de Biologia Experimental (FeSBE). Pesquisa financiada pelo CNPq através de Auxílio Individual (Processo: 477258/2001-8) e Bolsa de Produtividade em Pesquisa (Processo: 307182/2003-7).

2 Endereço para correspondência: Laboratório de Percepção, Neurociências e Comportamento, Depto. de Psicologia, Universidade da Paraíba, Campus I, Cidade Universitária. João Pessoa, PB. CEP 58051-900. E-mail: natanael.santos@pesquisador.cnpq.br. riais básicos decorrentes de estados patológicos (Akutsu \& Legge, 1995; Bour \& Apkarian, 1996; Elliott \& Situ, 1998; O`Donnell \& cols., 2002; Slaghuis \& Thompson, 2003). Em termos gerais, a FSC possibilita uma avaliação teórica e clínica do sistema visual humano (Adams \& Courage, 2002; John, 1997; Montés-Micó \& Ferrer-Blasco, 2001; Vleugels, van Nunen, Lafosse, Ketelaer \& Vandenbussche, 1998; Wilson, Levi, Maffei, Rovamo \& De Valois, 1990).

Um exemplo prático da importância da FSC de estímulos de frequências espaciais e radiais na avaliação da percepção visual ocorre quando uma criança que passa o dia na frente de um computador reclama de visão pobre. Nesse caso, a FSC avalia se o prejuízo é devido a alterações nas frequências baixas que dão o formato geral do objeto (algo como ver um objeto pouco iluminado à noite), a alterações nas frequências médias que dão o formato que se assemelha ao objeto final, a alterações nas frequências altas que são responsáveis pelo detalhes finos dos objetos (acuidade) ou, ainda, a alterações em mais de uma dessas faixas de frequências. É importante destacar que quanto mais cedo certas alterações são detectadas, melhor a intervenção e a recuperação.

\section{Detecção de Estímulos Radiais Circularmente Simétricos}

Em 1960, Kelly sugeriu a utilização de estímulos circularmente simétricos modulados pela função cilíndrica de Bessel 
de ordem zero $\left(\mathrm{J}_{0}\right)$. Essa classe de estímulos elementar tem sido denominada de frequência radial porque a modulação de contraste varia de acordo com o seno ou co-seno na direção radial, considerando o centro do círculo como a origem do sistema de coordenadas polares (ver Figura 1). Kelly partiu da ideia de que padrões radiais circularmente simétricos, definidos em coordenadas polares, poderiam ser mais apropriados para estudar o sistema visual humano devido à simetria aproximadamente circular da retina (Kelly, 1960) e mais naturais por fornecerem um centro claro de fixação (Kelly \& Magnuski, 1975). O mesmo não acontece com padrões definidos em coordenadas cartesianas, por exemplo, grade senoidal. Grade senoidal é um estímulo elementar cuja luminância varia de acordo com o seno ou co-seno, em uma direção no espaço, em um sistema de coordenadas cartesianas (Figura 1).

Em 1975, Kelly e Magnuski mensuraram a FSC para frequências radiais em adultos. O principal objetivo desses autores foi comparar a resposta de adultos para estímulos radiais e grades senoidais. Em 1982, Kelly replicou parte do estudo de 1975, com a técnica psicofísica de ajustamento de contraste, em que o participante ajustava seu próprio limiar de contraste. Os resultados obtidos por Kelly nos dois estudos com adultos demonstraram que a faixa de maior sensibilidade para estímulos radiais foi por volta de 0,5-2,0 cpg. Os resultados de Kelly também mostraram que o sistema visual era mais sensível para grades senoidais do que para estímulos radiais.

Já em 1997, Simas, Santos e Thiers mensuraram e compararam a sensibilidade ao contraste de adultos para padrões definidos matematicamente em coordenadas cartesianas (e.g., grades senoidais) e padrões definidos em coordenadas polares (e.g., estímulos radiais e angulares). Os resultados de Simas e colaboradores foram semelhantes aos resultados de Kelly (1982) e Kelly e Magnuski, (1975). Isto é, o sistema visual de adultos foi mais sensível para grades senoidais do que para estímulos radiais modulados pela função esférica de Bessel. Outros resultados e informações relacionadas à definição matemática e variação física de contraste em estímulos radiais podem ser encontrados em Simas e Santos (2002).

\section{Sensibilidade ao Contraste e Desenvolvimento}

A FSC começou a ser utilizada para avaliar as funções sensoriais básicas da visão de crianças a partir da década de 70 (Atkinson, Braddick \& Braddick, 1974; Atkinson, Braddick \& Moar, 1977). Desde então, a FSC tem sido descrita com técnicas psicofísicas e eletrofisiológicas utilizando estímulos elementares do tipo grades senoidais verticais (Allen, Tyler \& Norcia, 1996; Cannon JR, 1983; Norcia, Tyler \& Hamer, 1990; Peterzell, Werner \& Kaplan, 1995). Os principais achados com grades senoidais indicam que a FSC de recém-nascidos é muito pobre ao nascimento, melhorando rapidamente durante os primeiros meses (Allen \& cols., 1996; Atkinson \& cols., 1974, Atkinson \& cols., 1977; Cannon JR, 1983; Norcia \& cols., 1990; Peterzell \& cols., 1995). Esses estudos mostram ainda que o processo de maturação da FSC se estende além dos primeiros anos.
Os trabalhos com crianças a partir da idade pré-escolar demonstram que o processo de desenvolvimento da sensibilidade ao contraste para grade senoidal ou a faixa etária em que as crianças atingem a performance do adulto depende das condições experimentais e do método empregado para determinar a FSC (Adams \& Courage, 2002; Arundale, 1978; Benedek, Benedek, Kéri \& Janáky, 2003; Bradley \& Freeman, 1982; Ellemberg, Lewis, Liu \& Maurer, 1999). Por exemplo, Bradley e Freeman (1982) mensuraram a FSC com o método psicofísico da escolha forçada para crianças de 2 a 16 anos, utilizando grades senoidais e luminância média de $250 \mathrm{~cd} / \mathrm{m}^{2}$. Os resultados indicaram que as crianças atingem os níveis de sensibilidade ao contraste dos adultos aos 8 anos de idade. Estes pesquisadores relataram, ainda, que o método psicofísico da escolha forçada pode ser realizado com sucesso em crianças acima de 3,5 anos.

Ellemberg e cols. (1999) mediram a FSC com o método psicofísico dos limites para crianças de 4 a 7 anos e adultos, utilizando grandes senoidais e luminância média de 9 cd/ $\mathrm{m}^{2}$. Os autores não encontraram diferenças significativas na FSC entre crianças de 7 anos e adultos. Adams e Courage (2002) mediram a FSC com o método psicofísico do olhar preferencial para crianças de 1 mês a 9 anos, utilizando estímulos do tipo grade senoidal impressos em cartões e luminância média de $70 \mathrm{~cd} / \mathrm{m}^{2}$. Os resultados mostraram que a sensibilidade ao contraste das crianças desenvolve-se rapidamente até os três anos de idade; depois disso, continua desenvolvendo-se mais lentamente, atingindo, finalmente, níveis de sensibilidade ao contraste de adultos por volta dos 9 anos. Já Benedek e cols. (2003) mensuraram a FSC com o método do ajustamento em crianças e adolescentes de 5 a 15 anos, utilizando estímulos do tipo grade senoidal com dois níveis de luminância, 0,09 e $9 \mathrm{~cd} / \mathrm{m}^{2}$. Os dados mostraram que as crianças só atingiram a sensibilidade ao contraste do adulto por volta dos 11-12 anos.

No presente estudo, mediu-se curvas de sensibilidade ao contraste para grades senoidais (FSC) e para frequências radiais (FSCr) de crianças de 6 a 13 anos em nível baixo de luminância (luminância média de $0,7 \mathrm{~cd} / \mathrm{m}^{2}$ ), utilizando o método psicofísico da escolha forçada. As principais proposições para estudos dessa natureza vêm da necessidade de: (i) identificar que tipo de estímulo ou frequência espacial uma criança em uma determinada idade pode detectar; (ii) determinar qual o estímulo elementar mais adequado para caracterizar a resposta do sistema visual; (iii) estudar o desenvolvimento sensorial da percepção visual; (iv) investigar os mecanismos sensoriais envolvidos no processamento de padrões visuais espaciais; e (v) testar e aperfeiçoar uma técnica psicofísica capaz de caracterizar e acompanhar a resposta do sistema visual com e sem patologias.

O objetivo principal deste estudo foi determinar e comparar a resposta (sensibilidade ao contraste) do sistema visual para estímulos do tipo grade senoidal (FSC) e padrões radiais circularmente simétricos (FSCr). As pesquisas que determinaram a resposta do sistema visual humano para frequências radiais são dispersas e raras com adultos (Kelly, 1982; Kelly \& Magnuski, 1975; Santos, Oliveira, Nogueira \& Simas, 2006; Santos \& Simas, 2002; Simas, Nogueira \& Santos, 2005; Simas \& Santos, 2002). Além disso, não foi encontrado nenhum estudo na literatura comparando a 
resposta do sistema visual de crianças para estímulos elementares de frequências radiais (ou estímulos simétricos com configurações semelhantes) e grades senoidais.

\section{Método}

\section{Participantes}

Participaram do estudo 40 crianças de ambos os sexos. As mesmas foram distribuídas em quatro grupos (6-7 anos $(\mathrm{N}=10 ; \mathrm{M}=6,87 ; \mathrm{DP}=0,68)$, 8-9 anos $(\mathrm{N}=10 ; \mathrm{M}=8,74$; $\mathrm{DP}=0,77), 10-11$ anos $(\mathrm{N}=10 ; \mathrm{M}=10,73 ; \mathrm{DP}=0,46)$ e $12-13$ anos $(\mathrm{N}=10 ; \mathrm{M}=12,95 ; \mathrm{DP}=0,57))$. Os símbolos $M$ e $D P$ representam a idade média (ano, mês) e desvio padrão (mês) de cada grupo, respectivamente. Todos os participantes apresentavam acuidade visual normal ou corrigida e não tinham história de patologia ocular ou neurológica. A participação na pesquisa ocorreu mediante assinatura de termo de consentimento livre e esclarecido pelos responsáveis conforme a Resolução $n^{0}$ 196/96 do Conselho Nacional de Saúde (Ministério da Saúde), que trata das diretrizes e normas de pesquisas envolvendo seres humanos. O projeto do estudo foi aprovado pelo Comitê de Ética local (Centro de Ciências da Saúde, CCS), Processo 149/2005.

\section{Equipamentos e estímulos}

Os estímulos foram gerados em um monitor de vídeo CLINTON MEDICAL monocromático de 21 polegadas, digital e de alta resolução, controlado por um microcomputador. Uma cadeira foi fixada a $150 \mathrm{~cm}$ da tela do monitor de vídeo. A luminância média foi de $0,7 \mathrm{~cd} / \mathrm{m}^{2}$, ajustada por um fotômetro do tipo SPOT METTER, com precisão de um grau, ASAHI PENTAX. A luminância mínima foi de $0,5 \mathrm{~cd} /$ $\mathrm{m}^{2}$ e a máxima de $0,9 \mathrm{~cd} / \mathrm{m}^{2}$. O ambiente do laboratório era cinza para melhor controle da luminância.

Foram utilizados estímulos do tipo grade senoidal vertical e estímulos radiais com frequências espaciais de 0,25, 0,5, 1 e 2 ciclos por grau de ângulo visual (cpg). Esses estímulos eram circulares, com um diâmetro de 7 cpg a 150 cm de distância da tela, e foram gerados em tons de cinza e apresentados em tempo real no monitor (Figura 1).

\section{Procedimento}

As estimativas foram realizadas com o método psicofísico da escolha forçada (Santos \& cols., 2006; Santos \& Simas, 2002; Simas \& Santos, 2002; Wetherill \& Levitt, 1965). Esse método se baseia no cálculo da probabilidade de acertos consecutivos por parte do participante, ou seja, em cerca de 100 oportunidades de escolha entre dois estímulos, a frequência espacial (estímulo de teste) é percebida, por exemplo, em 79 oportunidades pelo voluntário. O procedimento para medir o limiar para cada frequência consistiu na apresentação sucessiva simples do par de estímulos, tendo o participante que escolher dentre eles qual continha a frequência espacial. O
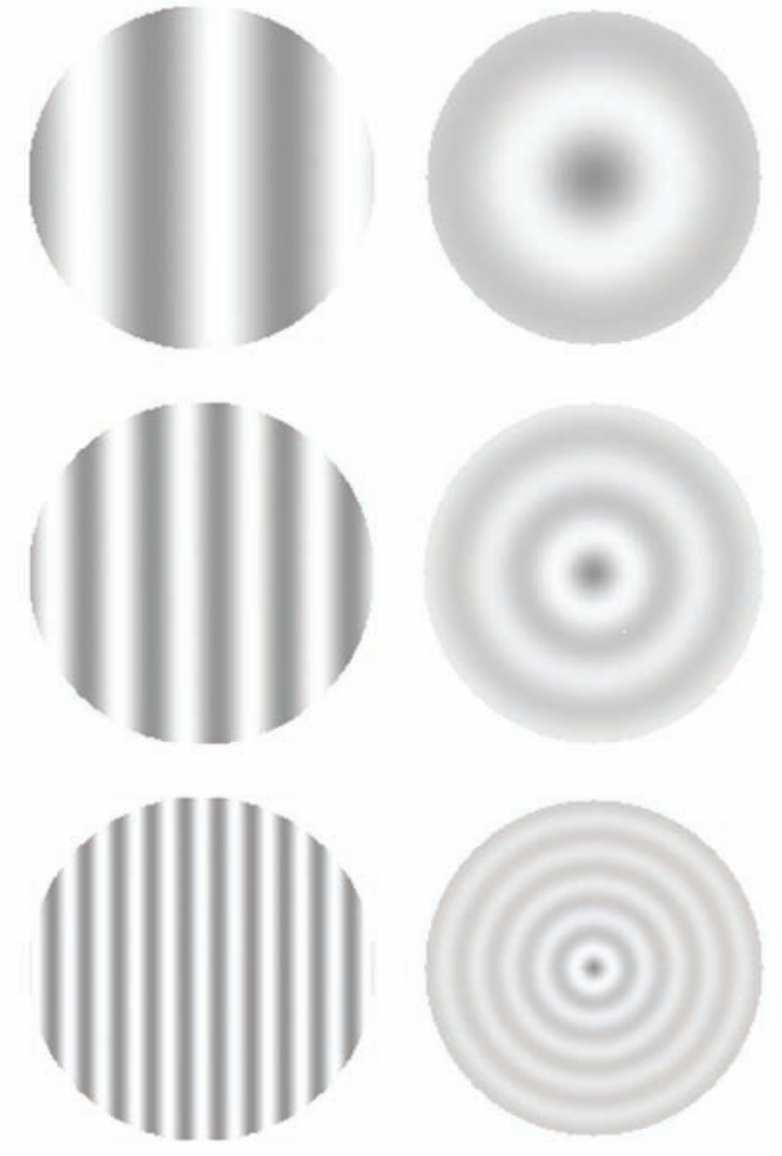

Figura 1. Exemplos de estímulos de freqüências espaciais (à esquerda) e radiais (à direita) de 0,5; 1,0 e 2,0 cpg, respectivamente, de cima para baixo. Estímulos originalmente calibrados para serem vistos a $150 \mathrm{~cm}$ de distância.

outro estímulo (neutro) foi sempre um padrão homogêneo com luminância média de $0,7 \mathrm{~cd} / \mathrm{m}^{2}$. O critério adotado para variar o contraste de cada frequência espacial testada foi o de três acertos consecutivos para decrescer uma unidade e um erro para acrescer a mesma unidade $(0,08 \%)$.

Durante cada sessão experimental foi apresentada uma sequência de estímulos. Essa sequência era iniciada com um sinal sonoro, o qual era acompanhado imediatamente pela apresentação do primeiro estímulo por $2 \mathrm{~s}$, seguido de um intervalo entre estímulos de $1 \mathrm{~s}$ e pela apresentação do segundo estímulo por $2 \mathrm{~s}$. Por fim, ocorria a resposta do participante. A ordem de apresentação dos estímulos era aleatória. Se a resposta do voluntário fosse correta, outro sinal sonoro ocorria, seguido por um intervalo de $3 \mathrm{~s}$, e a sequência era repetida. O sinal sonoro que indicava o início da apresentação do par de estímulos e o que indicava a escolha correta eram diferentes. A sessão experimental terminava automaticamente assim que o critério de cinco valores de contraste ou cinco reversões era atingido.

Cada um dos pontos (ou frequências) da curva de limiar de contraste para frequência espacial (FSC) e frequência radial (FSCr) foi estimado pelo menos duas vezes (duas sessões experimentais), em dias diferentes, por cada um dos participantes. No final dos experimentos, a sensibilidade ao contraste para os dois estímulos havia sido medida para cada criança, inde- 
pendentemente da idade. Em média, 20 curvas foram medidas para cada estímulo e cada uma das faixas etárias ou grupo de crianças (6-7, 8-9, 10-11 e 12-13 anos), totalizando 160 sessões experimentais por grupo, 80 para grades senoidais e 80 para frequências radiais. Todas as estimativas foram medidas à distância de 150 cm, com visão binocular.

Os participantes se sentaram individualmente em uma cadeira fixada a $150 \mathrm{~cm}$ da tela do monitor e foram instruídos a pressionar o botão do lado esquerdo do mouse quando o estímulo de teste (frequência espacial ou radial) era apresentado primeiro e o botão do lado direito quando era apresentado em segundo lugar, isto é, após o estímulo neutro. Os experimentos só começaram quando o experimentador certificou-se que a criança entendeu e respondeu conforme as instruções Essas instruções foram dadas em uma sessão de treino e ambientação às condições experimentais, até a criança discernir entre o estímulo com listras claro-escuras e o estímulo homogêneo em tom de cinza.

\section{Resultados}

Os valores de contrastes máximos e mínimos obtidos para cada frequência foram agrupados em planilhas por estímulos (grade senoidal ou radial) e por faixa etária (6-7,
8-9, 10-11 e 12-13 anos) e a grande média foi utilizada como estimativa da sensibilidade ao contraste que é o inverso do limiar de contraste.

As Figuras 2A, 2B, 2C e 2D mostram as curvas de sensibilidade ao contraste de frequências espaciais (FSC) e radiais (FSCr) para crianças de 6-7, 8-9, 10-11 e 12-13 anos, respectivamente. As frequências espaciais e radiais são apresentadas nos gráficos em função da sensibilidade ao contraste do sistema visual humano. As barras verticais indicam os erros padrão das médias com intervalos de 99\% de confiança, corrigidos para o tamanho da amostra pelo teste estatístico t-Student.

Os resultados mostraram que a sensibilidade máxima ocorreu em 0,5 cpg para as frequências espaciais (FSC) e em 0,25 cpg para as frequências radiais (FSCr) em todas as idades. Ainda, a sensibilidade ao contraste das crianças de 6-7, 8-9, 10-11 e 12-13 anos foi em média 2,4, 2,3, 2,1 e 2,1 vezes menor para as frequências radiais do que para as frequências espaciais, respectivamente. Estes valores médios foram obtidos pela média aritmética das diferenças na detecção de contraste dos dois estímulos para as quatro frequências testadas. Observa-se que a maior diferença média de sensibilidade ao contraste entre os dois estímulos ocorreu nas crianças de 6-7 anos (média de 2,4 vezes). Já a menor ocorreu entre as crianças de 10-11 e 12-13 anos (média de 2,1 vezes).
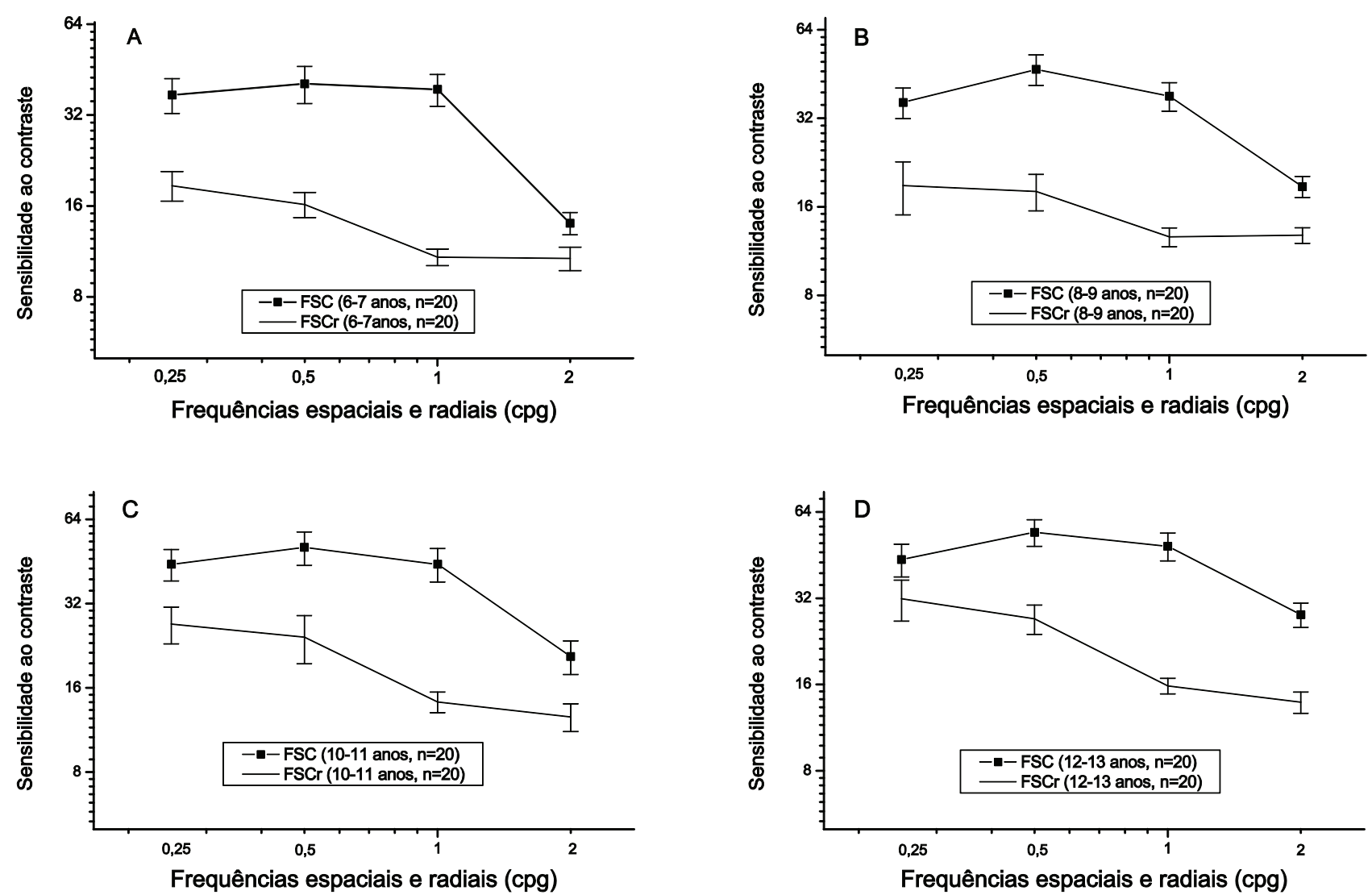

Figura 2. Curvas de sensibilidade ao contraste para frequências espaciais (--) e radiais $(-)$ de crianças de 6-7 anos (2A), 8-9 anos (2B), 10-11 anos (2C) e 12-13 anos (2D). O " $n$ " representa o número de curvas medidas para cada faixa etária. As linhas verticais mostram o erro padrão da média para cada frequência $(0,25 ; 0,5 ; 1$ e 2 cpg). 
A ANOVA para medidas repetidas revelou diferenças significativas entre as idades, $\left.F_{(3,620)}=147,2 ; \mathrm{p}<0,001\right)$; entre os estímulos, $\mathrm{F}_{(1,620)}=3994,9 ; \mathrm{p}<0$,001; entre as frequências, $\mathrm{F}_{(3,1860)}=1293,5 ; \mathrm{p}<0,001$; e interação significativa de estímulo versus idade, $\mathrm{F}_{(3,620)}=26,1 ; \mathrm{p}<0,001$; estímulo versus frequência, $F_{(3,1860)}=244,1 ; p<0,001$; e estímulo versus frequência versus idade, $\mathrm{F}_{(9,1860)}=19,8 ; \mathrm{p}<0,001$. As análises com o teste post-hoc Tukey HSD mostraram que as crianças de 6-7, 8-9, 10-11 e 12-13 anos apresentaram maior sensibilidade ao contraste para grades senoidais do que para estímulos radiais em todas as frequências $(\mathrm{p}<0,001)$.

\section{Discussão}

Em linhas gerais, os resultados sugerem que o sistema visual das crianças é mais sensível para grades senoidais (FSC) do que para estímulos radiais (FSCr) e que as diferenças entre as curvas de FSC e FSCr, em todas as faixas etárias, foram maiores nas frequências intermediárias. Esses resultados são consistentes com a existência de mecanismos distintos sensíveis a estímulos concêntricos circularmente simétricos em coordenadas polares.

Este estudo focaliza algumas questões básicas relacionadas ao processo de desenvolvimento visual de contraste. Por exemplo: (1) caracterizou a sensibilidade ao contraste para grade senoidal (FSC) e para frequência radial (FSCr) com o método psicofísico da escolha forçada; (2) determinou a faixa de máxima sensibilidade ao contraste para os dois estímulos em crianças de 6 a 13 anos; e (3) verificou para qual dos estímulos o sistema visual das crianças apresenta maior sensibilidade.

\section{Mensuração da FSC e FSCr de crianças com o método psicofísico da escolha forçada}

Os resultados mostram que o método psicofísico da escolha forçada, normalmente empregado nas pesquisas com adultos, pode ser utilizado de forma confiável para mensurar a FSC de crianças para estímulos do tipo grade senoidal e frequência radial. Observa-se que as curvas de sensibilidade ao contraste (FSC e FSCr) são consistentes uma vez que elas apresentam perfis gerais semelhantes e com as mesmas tendências em todas as faixas etárias (figuras 2A, 2B, $2 \mathrm{C}$ e 2D). $\mathrm{O}$ aspecto que se sobressai é a diferença entre as curvas de FSC e FSCr, o que provavelmente pode ser relacionado ao domínio da sensibilidade ao contraste do sistema visual que varia de acordo com as propriedades físicas dos estímulos (este aspecto será discutido mais a frente).

\section{Desenvolvimento da sensibilidade ao contraste de estímulos radial e grade senoidal}

Os resultados mostraram diferenças estatísticas entre as idades e uma interação entre estímulo versus idade. Isso significa que houve diferenças entre os quatros grupos, de forma que a sensibilidade média das crianças de 6-7 anos foi da ordem de 1,2 vezes menor do que a das crianças de
8-9 anos, que foi da ordem de 1,3 vezes menor do que a das crianças de 10-11 anos, que foi da ordem de 1,2 vezes menor do que a das crianças de 12-13 anos, tanto para frequências espaciais como para frequências radiais. No geral, estes resultados indicam que a FSC melhora gradativamente e de forma significante com o aumento da idade para ambos os estímulos. Estes resultados são semelhantes aos dados de Benedek e cols. (2003) que encontraram que a FSC de crianças se desenvolve até os 11-12 anos.

\section{Curvas de FSC e FSCr e faixas de máxima sensibilidade ao contraste}

Os resultados mostraram que a sensibilidade máxima de contraste em níveis mesópicos de luminâncias ocorreu na faixa de frequência de 0,5 cpg para grades senoidais e em 0,25 pcg para estímulos radiais, em todas as faixas etárias. Esses resultados com luminância mesópica $(0,7$ cd/ $\mathrm{m}^{2}$ ), comparados aos dados encontrados na literatura com luminância média acima de $9 \mathrm{~cd} / \mathrm{m}^{2}$ (Adams \& Courage, 2002; Bradley \& Freeman, 1982; Ellemberg \& cols., 1999; Kelly, 1982; Kelly \& Magnuski, 1975), mostram que a utilização de luminância baixa desloca a zona de máxima sensibilidade para a esquerda da FSC (i.e. 0,5 cpg) e da FSCr (i.e., 0,25 cpg). Isso porque em níveis de luminância alta, a faixa de máxima sensibilidade ocorre por volta de 3,0-5,0 cpg para grade senoidal (Adams \& Courage, 2002; Bradley \& Freeman, 1982; Ellemberg \& cols., 1999) e por volta de 0,5-2,0 cpg para frequências radiais (Kelly, 1982; Kelly \& Magnuski, 1975; Simas \& cols., 1997). Além disso, em níveis baixos de luminância, o sistema visual humano possivelmente não detecta frequências espaciais acima de 2,4 cpg (Benedek \& cols., 2003). Por causa desse fato, este trabalho se limitou a investigar frequências espaciais entre 0,25 e 2,0 cpg. O deslocamento da sensibilidade máxima para frequências mais baixas pode estar relacionado ao funcionamento da via visual magnocelular que é especializada no processamento de frequências espaciais baixas em níveis baixos de luminância (Benedek \& cols., 2003; Ellemberg \& cols., 1999). Em outras palavras, o processamento visual da forma e contraste envolve pelo menos dois sistemas: a via visual parvocelular, que é especializada no processamento de frequências espaciais médias e altas ou detalhes finos e opera em níveis altos ou fotópicos de luminância, e a via visual magnocelular, que é especializada no processamento de frequências espaciais baixas e opera em níveis baixos ou escotópicos de luminância.

\section{Sensibilidade ao contraste para estímulos radiais versus grades senoidais}

Os resultados mostraram que a sensibilidade ao contraste do sistema visual das crianças de 6-7, 8-9, 10-11 e 12-13 anos foram aproximadamente da ordem de duas vezes mais sensíveis para estímulos de grades senoidais do que para estímulos radiais (figuras 2A, 2B, 2C e 2D). As diferenças no processamento visual dos dois estímulos foram estatisticamente diferentes em todas as faixas etárias $(p<0,001)$. Essses 
achados já eram esperados, pois pesquisas com adultos já haviam demonstrado resultados semelhantes (Kelly, 1982; Kelly \& Magnuski, 1975; Simas \& Santos, 2002; Simas \& cols., 1997). Entretanto, este estudo mostrou que o sistema visual das crianças de 6-13 anos também é mais sensível para grades senoidais, FSC, do que para frequências radiais, FSCr. Essa redução na FSCr comparada a FSC pode estar relacionada à própria definição física dos estímulos. Isto é, grade senoidal é um estímulo visual elementar clássico cuja luminância mínima e máxima varia fisicamente em torno da luminância média, em uma direção no espaço, de acordo com o seno e/ou o co-seno, sendo facilmente definido em um sistema de coordenadas cartesianas. Ao passo que o estímulo visual de frequência radial é um padrão elementar cuja luminância mínima e máxima varia fisicamente em torno da luminância média na direção radial (ou seja, a luminância varia de acordo com o seno e/ou co-seno do centro para a periferia do estímulo), o que o torna um estímulo facilmente definido em um sistema de coordenadas polares com a origem do sistema de coordenadas no centro do estímulo (Kelly, 1982; Kelly \& Magnuski, 1975; Simas \& Santos, 2002). Os estímulos radiais apresentam contraste mais intenso no centro e menos intenso na periferia (Figura 1), tendendo a ser constante no infinito. Nesse contexto, é possível hipotetizar que a redução na FSCr comparada à FSC pode ser devida aos estímulos radiais não possuírem amplitude de contraste (ou distribuição de luminância) equivalente ao longo do estímulo, isto é, eles apresentam amplitude máxima no centro (fóvea), diminuindo para a periferia do campo visual (Simas \& Santos, 2002). Ao contrário, a amplitude da grade senoidal é equivalente ao longo de todo o estímulo ou campo visual (Figura 1). Então, é possível que a quantidade de canais ou a forma (intensidade) com que os canais específicos para cada padrão sejam ativados (na fóvea e próximo à fóvea) faça a diferença. A relação lógica por trás desse processo é que, se os dois padrões ativam canais específicos de formas diferentes, aquele que ativa mais intensamente o maior número de canais poderia apresentar maior sensibilidade.

Ao observar as curvas de FSC e FSCr das crianças de 6 a 13 anos (figuras 2A, 2B, 2C e 2D), parece que cada conjunto de estímulos de grade senoidal e radial tem atributos peculiares relacionados à detecção da forma e de contraste que os diferenciam entre si. As alterações na sensibilidade do sistema visual para esses estímulos são difíceis de serem explicadas por um mecanismo único. Essa hipótese pode ser reforçada por estudos psicofísicos (Santos \& Simas, 2002; Simas \& Santos, 2002; Simas \& cols., 1997; Wilson \& Wilkinson, 1998; Wilson, Wilkinson \& Asaad, 1997) e neurofisiológicos (Gallant, Connor, Rakshit, Lewis \& van Essen, 1996; Heywood, Gadotti \& Cowey, 1992; Merigan, 1996; Wilkinson \& cols., 2000) que sugerem que grade senoidal e padrão radial são processados por mecanismos e áreas visuais distintas. Por exemplo, Kobatake e Tanaka (1994) encontraram células seletivas a estímulos radiais na área visual V2, enquanto Gallant e cols. (1996) encontraram evidências para estímulos radiais na área visual V4. Já Wilkinson e cols. (2000), utilizando a técnica de imagem de ressonância magnética funcional, sugeriram o envolvimento de V4 e do córtex ínfero-temporal (IT) no processamento de padrões em coordenadas polares. Nesse contexto, é possível que as diferenças de sensibilidades ao contraste encontradas para estímulos do tipo grade senoidal definidas em coordenadas cartesianas e estímulos radiais definidos em coordenadas polares possam estar relacionadas ao envolvimento de vias ou áreas visuais diferentes.

\section{Considerações Finais}

Os resultados aqui obtidos demonstram que a habilidade que o sistema visual possui para detectar estímulos depende das características físicas que definem a modulação do contraste do estímulo. Essas características, por sua vez, podem estar relacionadas à detecção, ao processamento neural e à integração final da imagem percebida. Em linhas gerais, levando em conta os resultados com crianças de 6 a 13 anos do presente estudo e outros encontrados na literatura, podese inferir que o sistema visual utiliza mecanismos ou vias visuais distintas para processar padrões espaciais definidos em coordenadas cartesianas (e.g., grades senoidais) e padrões espaciais definidos em coordenadas polares (e.g., estímulos radiais). É nessa perspectiva que prosseguiremos com as nossas investigações, inclusive incluindo novas variáveis, como por exemplo, outros níveis de luminância.

\section{Referências}

Adams, R. J., \& Courage, M. L. (2002). Using a single test to measure human contrast sensitivity from early childhood to maturity. Vision Research, 42, 1205-1210.

Akutsu, H., \& Legge, G. E. (1995). Discrimination of compound gratings: Spatial-frequency channels or local features? Vision Research, 35, 2685-2695.

Allen, D., Tyler, C. W., \& Norcia, A. M. (1996). Development of grating acuity and contrast sensitivity in the central and peripheral visual field of the human infant. Vision Research, 36, 1945-1953.

Arundale, K. (1978). An investigation into the variation of human contrast sensitivity with age and ocular pathology. British Journal of Ophthalmology, 62, 213-215.

Atkinson, J., Braddick, O., \& Braddick, F. (1974). Acuity and contrast sensitivity of infant vision. Nature, 247, 403-404.

Atkinson, J., Braddick, O., \& Moar, K. (1977). Contrast sensitivity of the human infant for moving and static patterns. Vision Research, 17, 1045.1047.

Benedek, G., Benedek, K., Kéri, S., \& Janáky, M. (2003). The scotopic low-frequency spatial contrast sensitivity develops in children between the ages of 5 and 14 years. Neuroscience Letters, 345, 161-164.

Bour, L. J., \& Apkarian, P. (1996). Selective broad-band spatial frequency loss in contrast sensitivity functions. Investigative Ophthalmology \& Visual Science, 37, 2475-2484.

Bradley, A., \& Freeman, R. D. (1982). Contrast sensitivity in children. Vision Research, 22, 953-959.

Candy, T. R., Crowell, J. A., \& Banks, M. S. (1998). Optical, receptoral, and retinal constraints on foveal and peripheral vision in the human neonate. Vision Research, 38, 3857-3870.

Cannon JR, M. W. (1983). Contrast sensitivity: Psychophysical and evoked potential methods compared. Vision Research, 23, 87-95. 
Cornsweet, T. N. (1970). Visual perception. New York: Academic Press.

Ellemberg, D., Lewis, T. L., Liu, C. H., \& Maurer, D. (1999). Development of spatial and temporal vision during childhood. Vision Research, 39, 2325-2333.

Elliott, D. B., \& Situ, P. (1998). Visual acuity versus letter contrast sensitivity in early cataract. Vision Research, 38, 20472052.

Gallant, J. L., Connor, C. E., Rakshit, S., Lewis, J. W., \& van Essen, D. C. (1996). Neural responses to polar, hyperbolic, and cartesian gratings in area V4 of the macaque monkey. Journal of Neurophysiology, 76, 2718-2739.

Heywood, C. A., Gadotti, A., \& Cowey, A. (1992). Cortical area V4 and its role in the perception of color. Journal of Neuroscience, 12, 4056-4065.

John, R. S. (1997). Contrast detection and orientation discrimination thresholds associated with meridional amblyopia. Vision Research, 37, 1451-1457.

Kelly, D. H. (1960). J stimulus patterns for vision research. Journal of the Optical Society of America, 50, 1115-1116.

Kelly, D. H. (1982). Motion and vision: IV. Isotropic and anisotropic spatial response. Journal of the Optical Society of America, 72, 432-439.

Kelly, D. H., \& Magnuski, H. S. (1975). Pattern detection and the two dimensional Fourier transform: Circular targets. Vision Research, 15, 911-915.

Kobatake, E., \& Tanaka, K. (1994). Neuronal selectivities to complex object features in the ventral visual pathway of the macaque cerebral córtex. Journal of Neurophysiology, 71, 856-867.

Merigan, W. H. (1996). Basic visual capabilities and shape discrimination after lesions of extrastriate area V4 in macaques. Visual Neuroscience, 13, 51-60.

Montés-Micó, R., \& Ferrer-Blasco, T. (2001). Contrast sensitivity function in children: Normalized notation for the assessment and diagnosis of diseases. Documenta Ophthalmologica, 103, 175-186.

Norcia, A. M., Tyler, C. W., \& Hamer, R. D. (1990). Development of contrast sensitivity in the human infant. Vision Research, 30, 1475-1486.

O`Donnell, B. F., Nestor, P. G., Potts, G. F., Stylianopoulos, K. C., Shenton, M. E., \& McCarley, R. W. (2002). Spatial frequency discrimination in schizophrenia. Journal of Abnormal Psychology, 111, 620-625.

Peterzell, D. H., Werner, J., \& Kaplan, P. S. (1995). Individual differences in contrast sensitivity functions: Longitudinal study of 4-, 6- and 8-month-old human infants. Vision Research, 35, 961-979.

Santos, N. A., Oliveira, A. B., Nogueira, R. M. T. B. L., \& Simas, M. L. B. (2006). Mesopic radial frequency contrast sensitivity function for young and older adults. Brazilian Journal of Medical and Biological Research, 39, 791-794.
Santos, N. A., \& Simas, M. L. B. (2002). Percepção e processamento visual da forma em humanos: filtros de frequências radiais de 1 e 4 cpg. Psicologia: Reflexão \& Crítica, 15, 383-391.

Simas, M. L. B., Nogueira, R. M. T. B. L., \& Santos, N. A. (2005). Radial frequency stimuli and sine-wave gratings seem to be processed by distinct contrast brain mechanisms. Brazilian Journal of Medical and Biological Research, 38, 419-430.

Simas, M. L. B., \& Santos, N. A. (2002). Narrow-band 1, 2, 3, 4, 8, 16 and 24 cycles $/ 360^{\circ}$ angular frequency filters. Brazilian Journal of Medical and Biological Research, 35, 243-253.

Simas, M. L. B., Santos, N. A., \& Thiers, F. A. (1997). Contrast sensitivity to angular frequency stimuli is higher than that for sinewave gratings in the respective middle range. Brazilian Journal of Medical and Biological Research, 30, 633-636.

Slaghuis, W. L., \& Thompson, A. K. (2003). The effect of peripheral visual motion on focal contrast sensitivity in positiveand negative-symptom schizophrenia. Neuropsychologia, 41, 968-980.

Van Sluyters, R. C., Atkinson, M. S., Held, R. M., Hoffman, K., \& Shatz, C. J. (1990). The development of vision and visual perception. Em S. W. Spillmann \& J. S. Werner (Orgs.), Visual perception: The neurophysiological foundations (pp. 349-379). New York: Academic Press.

Vleugels, L., van Nunen, A., Lafosse, C., Ketelaer, P., \& Vandenbussche, E. (1998). Temporal and spatial resolution in foveal vision of multiple sclerosis patients. Vision Research, 38, 2987-2997.

Wetherill, G. B., \& Levitt, H. (1965). Sequential estimation of points on a psychometric function. The British Journal of Mathematical and Statistical Psychology, 48, 1-10.

Wilkinson, F., James, T. W., Wilson, H. R., Gati, J. S., Menon, E. S., \& Goodale, M. A. (2000). An fMRI study of the selective activation of human extrastriate form vision areas by radial and concentric gratings. Current Biology, 10, 1455-1458.

Wilson, H. R., Levi, D., Maffei, L., Rovamo, J., \& De Valois, R. (1990). The perception of form: Retina to striate cortex. Em S. W. Spillmann \& J. S. Werner (Orgs.), Visual perception: The neurophysiological foundation (pp. 231-271). New York: Academic Press.

Wilson, H. R., \& Wilkinson, F. (1998). Detection of global structure in glass patterns: Implications for form vision. Vision Research, 38, 2933-2947.

Wilson, R. W., Wilkinson, F., \& Asaad, W. (1997). Concentric orientation summation in human form vision. Vision Research, 37, 2325-2330. 